\title{
Dual chamber pacing: how many patients remain in DDD mode over the long term?
}

\author{
Bassem Ibrahim, John E Sanderson, Barbara Wright, Richard Palmer
}

\begin{abstract}
Objective-DDD pacing is better than VVI pacing in complete heart block and sick sinus syndrome but is more expensive and demanding. In addition, some patients have to be programmed out of DDD mode and this may have an important impact on the cost-effectiveness of DDD pacing. The purpose of this study was to determine how many patients remain in DDD mode over the long term (up to 10 years).
\end{abstract}

Design-A retrospective analysis of the outcome over 10 years of consecutive patients who had their pacemakers programmed initially in DDD mode.

Setting-A district general hospital.

Patients-249 patients with DDD pacemakers. Sixty two patients $(24.9 \%)$ had predominantly sick sinus syndrome and $180(72 \cdot 3 \%)$ had predominantly atrioventricular conduction disease. Mean (range) complete follow up for this group of patients was 32 months (1-10 years). Results-Cumulative survival of DDD mode was $83 \cdot 5 \%$ at 60 months. Atrial fibrillation was the commonest reason for abandonment of DDD pacing. Atrial fibrillation developed in 30 patients $(12 \%)$, with atrial flutter in three $(1 \cdot 2 \%)$. Loss of atrial sensing or pacing, pacemaker mediated tachycardia, and various other reasons accounted for reprogramming out of DDD mode in eight patients $(3 \cdot 2 \%)$. Overall, an atrial pacing mode was maintained in $91 \%$ and VVI pacing was needed in only $9 \%$.

Conclusions-With careful use of programming facilities and appropriate secondary intervention, most patients with dual chamber pacemakers can be maintained successfully in DDD or an alternative atrial pacing mode until elective replacement, although atrial arrhythmia remains a significant problem. There are no good reasons, other than cost, for not using dual chamber pacing routinely as suggested by recent guidelines and this policy can be achieved successfully in a district general hospital pacing centre.

(Br Heart f 1995;74:76-79)

Keywords: dual chamber pacemaker; DDD mode; atrial fibrillation
Dual chamber atrioventricular synchronous pacing (DDD) has been shown in many studies to provide better haemodynamics and quality of life than ventricular inhibited single chamber pacing (VVI) in patients with complete atrioventricular block. $^{1-4}$ Nevertheless, the unreliability of some atrial leads, extra time required for atrial lead fixation, complexity of follow up, and high price of DDD units have created a reluctance to implant dual chamber pacemakers and VVI pacing has been recommended as acceptable treatment. ${ }^{5}$ Furthermore, patients occasionally need to be programmed out of DDD mode with loss of all the advantages of atrioventricular synchrony, and the frequency of this over the long term remains uncertain. This aspect may have an important influence on the assessment of the cost-effectiveness of DDD pacemakers. Gross et $a l^{6}$ found in their retrospective study in the United States that over a mean follow up of 33 months $18 \%$ of patients had their pacemaker permanently, and $2 \%$ temporarily, reprogrammed out of DDD mode and the DDD mode survival rate was $78 \%$ at 5 years. The major cause was the development of atrial fibrillation which was associated with dominant sinoatrial disease. Detollenaere $\mathrm{et}^{\mathrm{al}} \mathrm{l}^{7}$ from Belgium found that during a mean follow up of 30 months reprogramming to VVI mode was necessary in $15.5 \%$, with atrial fibrillation and flutter being the most common causes. ${ }^{7}$ There are little data from a United Kingdom centre in which the patient population may be different and in particular from a district general hospital where most pacemakers are now implanted. We have therefore reviewed our experience with dual chamber pacemakers to determine the acute and long-term outcome of a cohort of patients with DDD pacemakers, in particular to find out how many remain in DDD mode and what are the main reasons for loss of this mode.

Patients and methods

All available and completed notes of patients fitted with dual chamber pacemakers and followed in our pacemaker clinic were reviewed in this retrospective study. The implantations took place between September 1981 and April 1992. More than $95 \%$ of the implantations were carried out by two cardiologists. We used passive fixation atrial and ventricular 
Figure 1 Cumulative survival of the DDD pacing mode by the Kaplan-Meier method. The number of patients at each interval is shown.

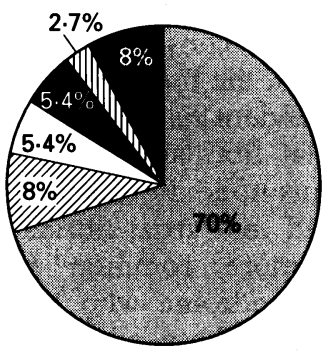

$A F$

Atrial flutter PMT

Failure of atrial pacing

血 Failure of atrial sensing

Miscellaneous

Figure 2 Causes of DDD mode abandonment and their relative incidence. $A F$, atrial fibrillation, $P M T$, pacemaker mediated tachycardia.

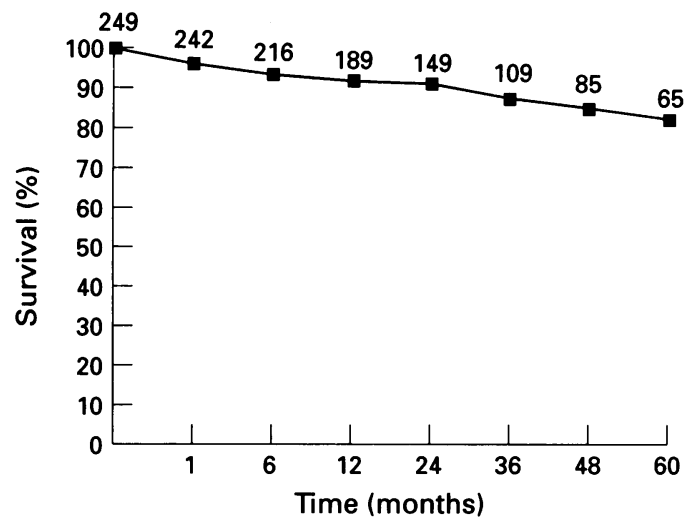

electrodes. They were inserted via the transvenous route using the left subclavian vein $(90 \%)$ and, to a less extent, the left cephalic vein.

Follow up visits were arranged at 1 and 3 months after implantation, and at 6-9 month intervals thereafter. Routine assessments included sensing and pacing thresholds on both leads, testing for pectoral muscle stimulation and myopotential inhibition, as well as checking the wound for signs of infection. Demographic information included patient age, gender, and indication for implantation,- - that is, dominant sinoatrial or atrioventricular conduction disease. The following information was also analysed: (a) duration of follow up; (b) need for secondary surgical intervention and its reason; (c) need for reprogramming out of DDD mode; and (d) reason for reprogramming including the development of atrial fibrillation, loss of atrial sensing or pacing, pacemaker mediated tachycardia, and lead dislodgment. Loss of atrial sensing or pacing without obvious dislodgment in $x$ ray film was still considered an indication for surgical exploration and repositioning of the lead.

\section{STATISTICAL ANALYSIS}

A cumulative survival curve of the DDD pacing mode was calculated using the Kaplan-Meier method. A pacemaker was defined as a nonsurvivor if it was programmed out of DDD mode for the rest of the patient's follow up.

\section{Results}

\section{PATIENT CHARACTERISTICS}

The notes of 249 patients fitted with dual chamber pacemakers who had their pacemakers programmed from the onset in DDD mode were available. The mean (SD) age of the 249 patients was 71 (10) years; $58 \cdot 2 \%$ were male. Sixty two patients $(24.9 \%)$ had predominant sinus node disease. Of 180 patients $(72 \cdot 3 \%)$ with predominant atrioventricular conduction disease, 140 had complete atrioventricular block and 40 were paced because of symptomatic $2: 1$ atrioventricular block or trifasicular block. Seven patients $(2 \cdot 8 \%)$ were paced because of carotid sinus syndrome, malignant vasovagal syndrome, and miscellaneous indications. No patient had documented atrial fibrillation before implantation. The mean (range) follow up was about 32 months (1-10 years).

\section{SECONDARY INTERVENTION}

Twenty four patients $(9.6 \%)$ needed reoperation during the follow up period. In total 12 atrial and two ventricular electrodes were repositioned to restore adequate sensing or pacing, or both, even in the absence of gross dislodgment. Eight atrial electrodes needed replacement; three because of failure of pacing because of high threshold, three as a result of fractures, and one each for an insulation break and loss of atrial sensing secondary to random component failure. One ventricular electrode was replaced because of insulation break and one for failure of pacing because of exit block. Two patients $(0 \cdot 8 \%)$ were reoperated on because of pacemaker infection. Pulse generator replacement for normal battery depletion was not considered a reparative intervention.

\section{PACEMAKER SURVIVAL IN DDD MODE}

In total 37 patients $(14.9 \%)$ required permanent programming out of DDD mode during the whole of the follow up period. At first follow-up visit, 1 month after implantation, $242(97 \cdot 2 \%)$ of 249 patients were still in DDD mode. After 6 months of follow up $94 \%$ of pacemakers remained in DDD, at 12 months $92 \cdot 1 \%$, at 24 months $91.9 \%$, at 36 months $88 \%$, at 48 months $85.8 \%$, and at 60 months $83.5 \%$ of pacemakers were still functioning in the DDD mode (fig 1).

\section{REASONS FOR REPROGRAMMING}

The development of atrial fibrillation was the most common indication for abandonment of DDD mode (fig 2). It was noted in 30 $(12.0 \%)$ of the 249 study patients. Atrial fibrillation developed in $10(16 \%)$ of 62 patients with sick sinus syndrome and $18(10 \%)$ of 180 with atrioventricular block (not significant; $P=0 \cdot 2$ ). Four patients had a single episode of atrial fibrillation, returned to sinus rhythm after treatment with amiodarone and were reprogrammed back to DDD mode (cardioversion was not used in any patient). Thirteen patients developed chronic atrial fibrillation and were programmed to VVI. In the remaining 13 patients, their atrial fibrillation was paroxysmal; nine were changed to DVI and four to DDI mode (fig 3). Atrial flutter developed in another three patients (programmed to VVI).

Pacemaker mediated tachycardia was the reason for abandonment of DDD in two patients. Two patients had their device programmed out of the DDD mode because of failure of atrial pacing. Pacemaker reprogramming was performed because of failure of atrial sensing in only one patient; one patient programmed into AAI because of high ventricular threshold and in another two patients for miscellaneous reasons. Thus, an atrial pacing mode (DDD, DVI, DDI, or AAI) was maintained in $91 \%$ and VVI pacing was required in only $9 \%$ (23 patients). 
Figure 3 Schematic representation of the incidence of atrial fibrillation $(A F)$ in this study population.

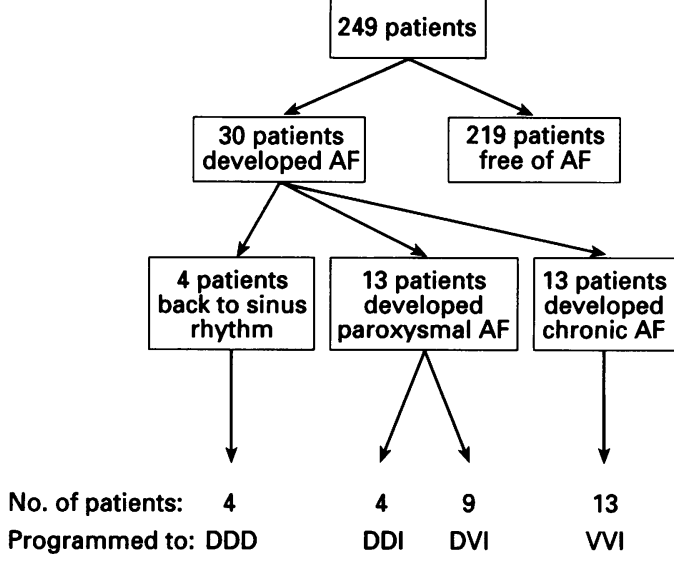

Discussion

Several studies have documented the advantages of DDD over VVI pacing in both atrioventricular block and sick sinus syndrome. ${ }^{1-4}$ VVI pacing is associated with an increase in cardiothoracic ratio, ${ }^{8}$ frequency of atrial fibrillation, ${ }^{9}$ higher incidence of thromboembolism, ${ }^{10}$ increase in plasma atrial natriuretic peptide, ${ }^{11}$ poorer exercise capacity, ${ }^{34}$ reduced quality of life, ${ }^{3412}$ and probably higher mortality. ${ }^{13}$ In patients with chronic symptomatic sinus node dysfunction and congestive heart failure survival is negatively affected by VVI pacing, while atrial synchronous pacing improves survival rate in this subset of patients. ${ }^{14}$

DDDR pacing may have additional advantages for patients with atrioventricular block and atrial chronotropic incompetence ${ }^{15} 16$ but this mode is still rarely used in the United Kingdom. Despite the considerable evidence that DDD (or DDDR) pacing is superior to ventricular pacing in atrioventricular block and sick sinus syndrome VVI units are still the most popular units implanted in the United Kingdom. Indeed, the use of VVI pacing has been defended. ${ }^{517}$ The arguments against using DDD pacing routinely for atrioventricular block include the expense, shorter life span, and more complicated follow up and programming. Furthermore if atrioventricular synchrony is lost because circumstances force a change to VVI mode then the patient will be left with a more costly unit from which he will derive little benefit. The question therefore is how often this occurs and what are the main causes. This is an important aspect of this cost-benefit equation. Published data are sparse. Earlier reports using older generators are no longer applicable to current pacemakers. Gross et $a l^{6}$ in the United States of America found that $78 \%$ of their patients were still in DDD mode at 5 years and our results are similar with $83.5 \%$ in DDD mode at 60 months. In addition, of the 37 patients in whom DDD mode was permanently abandoned, eight $(22 \%)$ were converted to other atrial sensing modes; one to AAI and seven to DDI. In comparison with the study by Gross et $a l,{ }^{6}$ however, we had a higher incidence of secondary surgical intervention-that is, $\mathbf{9 \cdot 6 \%}$ in the present study versus $4 \%$. Half of our reoperations were to reposition atrial elec- trodes. These were carried out despite the absence of radiological evidence of gross dislodgment in most cases. When faced with inadequate functioning of the atrial leadnon-sensing or non-pacing, or both-the choice was between reprogramming to VVI with loss of all the advantages of the DDD system and exploratory intervention. We usually opted for the latter to correct "microdislodgment" of the atrial electrode-that is, not seen in $x$ ray film, and in occasional cases to replace a faulty electrode due to fracture or insulation break. In the majority this policy led to the restoration of adequate sensing and pacing. This may account for the fact that loss of atrial sensing accounted for only $2.7 \%$ of permanent failures of DDD mode, as compared with $26.2 \%$ in the study of Gross $e t a l^{6}$ and perhaps contributes to the higher percentage of patients who remained in DDD mode $(83.5 \% v 78 \%)$.

Atrial fibrillation, which developed in $12 \%$ of our patients, was the most common reason for abandonment of DDD mode. This observation is in concordance with other studies. Gross $e t a l^{6}$ found that atrial fibrillation, whether chronic or paroxysmal, developed in $10 \%$ of the patients and accounted for $48 \%$ of reprogramming out of DDD mode. Similarly, chronic atrial fibrillation was responsible for $73 \%$ of loss of atrioventricular synchronous pacing in the study of Byrd et al. ${ }^{8}$ In a recently published study from a United Kingdom centre, however, Chamberlain-Webber $e^{2} a^{18}$ reported a low incidence of atrial fibrillation (3.3\%) in a group of 771 patients followed up over a mean (SD) of 40 (31) months. Detollenaere $e t \mathrm{al}^{7}$ found a high frequency of atrial flutter in their group, nearly half of the patients who developed atrial arrhythmia. In our study atrial flutter occurred in only three patients $(1 \cdot 2 \%)$, a rate similar to that found by Gross et al. ${ }^{6}$ Patients with sick sinus syndrome, however, had a 1.5 times greater risk of developing atrial fibrillation than patients with atrioventricular conduction defect $(16.1 \%$ v $10 \%$; although this was not statistically significant), and this is consistent with other studies. ${ }^{6-9}$ In addition, the mode of pacing seems to be an independent risk factor for the development of atrial fibrillation, especially in patients with sinus node dysfunction. Langenfeld et $a l^{19}$ showed that atrial fibrillation was significantly more common in patients with sick sinus syndrome who had VVI pacing compared with those who had DDD $(37 \% v 19 \%$ respectively, $\mathrm{P}<0.01)$. Similarly, Hesselson $e t$ al $l^{9}$ found in a group of 950 patients that $14 \%$ developed atrial fibrillation and that this was over four times more common in those with a VVI pacemaker, especially in those with sick sinus syndrome. In many patients, DDD modes can be maintained by cardioversion to sinus rhythm, either electrically or by drugs, followed by suppressive treatment with amiodarone or sotalol and this was our policy. The DDI mode is useful for paroxysmal atrial fibrillation. ${ }^{20}$ DDI mode provides atrial ventricular sequential pacing with atrial sensing, but no tracking during sinus rhythm. Atrial sensing 
prevents competitive pacing in the atrium and during an atrial tachyarrhythmia there is no possibility of inappropriate tracking. DDDR pacing may help to reduce the incidence of atrial fibrillation especially in those with atrioventricular block and chronotropic incompetence who in effect have sick sinus syndrome and who are more prone to develop atrial fibrillation. However, this is unproved. It is known that DDDR is preferable to VVIR for patients with chronotropic incompetence ${ }^{21}$ but not if DDDR is better than DDD alone with respect to the development of atrial fibrillation over the long term. Some DDDR pacemakers contain algorithms that limit inappropriate ventricular pacing rate during tracking of pathological supraventricular tachyarrhythmia and atrial flutter by Wenckebachlike prolongation of the atrioventricular interval. This may occasionally cause an unexpected extension of the atrioventricular interval in patients with high atrial rate and intact atrioventricular nodal conduction which may appear as failure of ventricular pacing on the electrocardiogram. ${ }^{22}$ Another possibility in the future for the management of atrial fibrillation is the incorporation of a mechanism for atrial defibrillation into a DDDR pacemaker. ${ }^{23}$

Another aspect of this study group which may be different is that we have used almost exclusively passive fixation atrial leads. $P$ wave amplitudes are initially better with passive fixation electrodes but chronically there is little difference, ${ }^{24}$ and atrial threshold stimulation energies do not differ between the two lead groups. There is no good evidence at the moment to support the notion that passive fixation atrial leads are associated with lower rates of atrial fibrillation but it is a possibility.

Pacemaker mediated tachycardia was a problem with the first generation DDD pacing systems but this is not so with the recent models. In our study, pacemaker mediated tachycardia accounted for only $5 \cdot 4 \%$ of the reasons for reprogramming out of DDD mode, and this was with the earlier models which had limited programmability and fixed post ventricular atrial refractory period.

The study is limited by its retrospective nature. There was no control group in whom results of VVI pacing were compared with those of DDD. Not all risk factors for the development of atrial fibrillation were routinely recorded. The number of patients included and the long duration of follow up, however, all support the validity of our data and their applicability to the general pacemaker population. Another aspect of this study relevant to practice in the United Kingdom is that it confirms the feasibility of a policy to insert DDD pacemakers where indicated in a district general hospital with good results. There is no reason for the newer pacing centres in these hospitals merely to implant VVI pacemakers.

\section{Conclusion}

In this study $83.5 \%$ of patients with dual chamber pacing systems were maintained in DDD pacing mode for at least 5 years, and $91 \%$ maintained some form of atrial pacing. The major reason (in $78 \%$ ) for abandoning DDD mode was the development of atrial fibrillation or atrial flutter. This continues to be a significant drawback and further research is required to identify those at greater risk of developing atrial tachyarrhythmia.

1 Kruse I, Ryden L. Comparison of physical work capacity and systolic time intervals with ventricular inhibited and atrial synchronous ventricular inhibited pacing. $\mathrm{Br}$ Heart f 1981;46:129-36.

2 Kruse I, Arnman K, Conradson TB, et al. A comparison of the acute and long-term hemodynamic effects of ventricular inhibited and atrial synchronous ventricular inhibited pacing. Circulation 1982;65:846-55.

3 Perrins EJ, Morley CA, Chan SC, Sutton R. Randomised control trial of physiological and ventricular pacing. $\mathrm{Br}$ Heart f 1983;50:112-7.

4 Rediker DE, Eagle KA, Homma S, Gillam LD, Harthorne JW. Clinical and haemodynamic comparison of VVI versus DDD pacing in patients with DDD pacemakers. Am $₹$ Cardiol 1988;61:323-9.

5 Petch MC. Who needs dual chamber pacing? BMF 1993; 307:215-6.

6 Gross JN, Moser S, Bendek ZM, Andrews C, Furman S. DDD pacing mode survival in patients with a dualchamber pacemaker. $f$ Am Coll Cardiol 1992;19: chamber

7 Detollenaere $\mathbf{M}$, van Wassenhove E, Jordaens L. Atrial arrhythmias in dual chamber pacing and their influence on long-term mortality. $P A C E$ 1992;15:1846-50.

8 Byrd CL, Schwartz SJ, Gonzales M, et al. DDD pacemakers maximize haemodynamic benefits and minimize complications for most patients. PACE 1988;11:1911-6.

9 Hesselson AB, Parsonnet V, Bernstein AD, Bonavita GJ. Deleterious effects of long term single chamber ventricular pacing in patients with sick sinus syndrome: the hidden benefits of dual chamber pacing. $₹ \mathrm{Am}$ Coll Cardiol den benefits of dual

10 Sutton R, Kenny RA. The natural history of sick sinus syndrome. $P A C E$ 1986;9:110-4

11 Stangl K, Weil J, Seitz K, et al. Influence of A-V synchrony on the plasma level of atrial naturetic peptide in patients with total A-V block. PACE 1988;11:1176-81.

12 Linde-Edelstam C, Norlander R, Unden AC, Orth-Gomer $\mathrm{K}$, Ryden L. Quality-of-life in patients treated with atrioventricular synchronous pacing compared to rate modulated ventricular pacing: a long-term, double-blinded, crossover study. PACE 1992;15:1467-76.

13 Linde-Edelstam C, Gullberg B, Norlander R, Pehrsson SK, Rosenqvist M, Ryden L. Longevity in patients with high degree atrioventricular block paced in the atrial synhigh degree atrioventricular block paced in the atrial synchronous or the fixed rate

14 Alpert MA, Curtis J, Sanfelippo JF, et al. Comparative survival following permanent ventricular and dualchamber pacing for patients with chronic symptomatic sinus node dysfunction with and without congestive heart failure. Am Heart $\mathcal{F}$ 1987;113:958-65.

15 Sutton R. DDDR pacing. PACE 1990;13:385.

16 Daubert C, Mabo PH, Pouillot CH, Lelong B. Atrial chronotropic incompetence. Implications for DDDR pacing. In: Barold SS, Mugica J, eds. New perspectives in pacing. In: Barold SS, Mugica J, eds. New perspectives in
cardiac pacing 2. Mt Kisco, New York: Futura cardiac pacing 2. M
Publishing 1991:251.

17 Ray SG, Griffith MJ, Jamieson S, Bexton RS, Gold RG. Impact of the recommendations of the British Pacing and Electrophysiology Group on pacemaker prescription and on the immediate costs of pacing in the Northern region. Br Heart $\mathcal{1} 1992 ; 68: 531-4$

18 Chamberlain-Webber R, Peterson MEV, Ingram A, Briers L, Sulton R. Reasons for reprogramming dual chamber pacemakers to VVI mode: a retrospective review using a computer database. PACE 1994;17:1730-6.

19 Langenfeld $\mathrm{H}$, Grimm W, Maisch B, et al. Atrial fibrillation and embolic complications in paced patients. PACE 1988;11:1667-72.

20 Irwin M, Harris L, Cameron D, Louis C, Radvanszky E, Goldman B. DDI pacing: indications, expectations, and follow-up. PACE 1994;17:274-9.

21 Landzberg JS, Franklin JO, Mahawar SK, et al. Benefits of physiologic atrioventricular synchronization for pacing with an exercise rate response. Am f Cardiol 1990;66: 193-7.

22 Leung SK, Lau CP, Leung WH, Tai YT, Chung F, Chow YH. Apparent extension of the atrioventricular interval due to sensor-based algorithm against supraventricular tachyarrhythmias. PACE 1994;17:321-30.

23 Keane D, Sulke N, Cooke R, et al. Endocardial cardioversion of atrial flutter and fibrillation [abstract]. $P A C E$ 1993;16:928.

24 Shandling AH, Castellanet MJ, Thomas LA, Messenger SC. The influence of endocardial electrode fixation status on acute and chronic atrial stimulation threshold and atrial endocardial electrogram amplitude. PACE 1990; 13:1116-22. 